\title{
Laparoscopic repair of abdominal incisional hernia
}

\author{
Xue-Fei Yang ${ }^{1}$, Jia-Lin Liu ${ }^{2}$ \\ ${ }^{1}$ Department of Surgery, the University of Hong Kong-Shenzhen Hospital, the University of Hong Kong, Shenzhen 518053, China; ${ }^{2}$ Department of \\ Hepatobiliary Surgery, Shenzhen People's Hospital \& Second Clinical Medical College of Jinan University, Shenzhen 518020, China \\ Correspondence to: Jia-Lin Liu. Department of Hepatobiliary Surgery, Shenzhen People's Hospital, No. 1017, North Dongmen Road, Luohu District, \\ Shenzhen 518020, China. Email: szliujialin@126.com or 4683755@qq.com.
}

\begin{abstract}
Abdominal incisional hernia is a common complication after open abdominal operations. Laparoscopic procedures have obvious mini-invasive advantages for surgical treatment of abdominal incisional hernia, especially to cases with big hernia defect. Laparoscopic repair of incisional hernia has routine mode but the actual operations will be various according to the condition of every hernia. Key points of these operations include design of the position of trocars, closure of defects and fixation of meshes. The details of these issues and experiences of perioperative evaluation and treatment will be talked about in this article.
\end{abstract}

Keywords: Incisional hernia; laparoscopic repair; mesh; fixation

Submitted Jul 19, 2016. Accepted for publication Jul 21, 2016.

doi: $10.21037 / \mathrm{atm} .2016 .09 .13$

View this article at: http://dx.doi.org/10.21037/atm.2016.09.13

\section{Introduction}

The incisional abdominal wall hernia is a type of hernia in which abdominal tissue or organs protrude through the incompletely healed fascia or muscularis of an abdominal incisional area due to intra-abdominal pressure. A common complication after abdominal surgery with an incidence rate of approximately $2-18 \%$, its occurrence is associated with wound infection, surgical mishandling, increased intra-abdominal pressure and other systemic factors such as smoking, malnutrition, jaundice, obesity, steroid use and depressed immunity. The probability of an abdominal incisional hernia occurring is significantly higher for longitudinal incisions than transverse incisions. Appropriate treatments are recommended (non-surgical/surgical treatments) $(1,2)$ because incisional abdominal wall hernias will not heal spontaneously.

\section{Indications}

According to the "Guidelines on the treatment of incisional abdominal wall hernia (2014 Edition)" published by the Hernia and Abdominal Wall Surgery Group, Chinese Medical Association, in 2014, the indications for incisional abdominal wall hernia treatment are listed as follows: (I) the diagnosis is confirmed; surgical assessment indicates low surgical risk; and patients are willing to pay the medical expenses for the hernia repair surgery; (II) the diagnosis is confirmed; surgical assessment indicates high surgical risk; and patients are required to complete the appropriate preoperative preparation, such as pulmonary rehabilitation and abdominal capacity expansion (artificial pneumoperitoneum); (III) the multidisciplinary treatment mode is recommended in patients with massive incisional hernia and loss of abdominal domain; the surgery plan is ideally determined by a multi-disciplinary team of specialists in plastic surgery, pulmonary medicine and intensive care; and (IV) the diagnosis is confirmed, and the hernia support belt is recommended to limit the progression of the incisional hernia in patients who are not indicated for surgery or who desire to delay the surgery.

\section{Contraindications}

* Patients who cannot tolerate general anesthesia and artificial pneumoperitoneum;

* Patients with acute incarcerated hernia accompanied by acute intestinal strangulation;

* Patients with an evident infectious lesion of the skin of 
Table 1 Classification of abdominal wall defects and respective surgical plans

\begin{tabular}{|c|c|c|}
\hline Classification & Classification parameter & Surgical approach selection \\
\hline $\begin{array}{l}\text { Moderate-sized } \\
\text { incisional hernia }\end{array}$ & $\begin{array}{l}\text { Maximum diameter of abdominal wall defect is } \\
\text { between } 4 \text { and } 8 \mathrm{~cm}\end{array}$ & $\begin{array}{l}\text { Abdominal wall defect is first closed, after which a mesh is placed to } \\
\text { cover the defect with mesh margins exceeding the defect by } 3-5 \mathrm{~cm} \\
\text { on both sides }\end{array}$ \\
\hline $\begin{array}{l}\text { Massive incisional } \\
\text { hernia }\end{array}$ & $\begin{array}{l}\text { Maximum diameter of abdominal wall defect is } \\
\text { greater than } 12 \mathrm{~cm} \text {, or the ratio of the volume of } \\
\text { the sac to the abdominal volume is greater than } \\
15 \% \text { (regardless of the abdominal wall defect } \\
\text { maximum diameter) }\end{array}$ & $\begin{array}{l}\text { Same as for moderate-sized, however, preoperative abdominal } \\
\text { peritoneal expansion should be conducted and abdominal muscle } \\
\text { compliance training should be performed for } 2 \text { to } 3 \text { weeks (the return } \\
\text { of hernia contents to the abdominal cavity plus the application of a } \\
\text { hernia support belt or progressive artificial pneumoperitoneum) }\end{array}$ \\
\hline
\end{tabular}

the surgical field or in the abdomen;

- Patients with previous comorbidities such as systemic endocrine, cardiovascular and respiratory diseases that are not ideally controlled, or in whom immunity is depressed, for example, in AIDS patients;

* Patients who present with tumor relapse or metastasis after surgery for abdominal malignant disease;

* Patients whose treatment hospital lacks an intensive care unit (ICU).

\section{Classification}

* Abdominal wall defects are divided into four categories based on size; the surgical plans for them are listed as Table 1;

* Based on the location of the abdominal wall defect, incisional hernias are divided as follows: (I) incisional hernia (including hernia above or below the umbilical incision or hernia above or below the para-umbilical incision) located in the anterior abdominal wall central region (along the midline or near the midline expanse); (II) incisional hernia (incisional hernia along the regions below the xiphoid process, above the pubis, below the costal margin and near the inguinal region) located in the anterior abdominal wall edge region; and (III) lateral abdominal wall hernia and posterior abdominal wall hernia (incisional hernia along the region between the ribs and the iliac or lumbar incisional hernia);

* According to the hernia recurrence frequency, the incisional hernias are divided into primary and recurrent incisional hernia.

\section{Surgical timing and approach}

Performance of the repair surgery is recommended for patients with incisional hernia after clinical observation and follow-up for more than 3 months following the healing of the incision, provided no infectious incision complications have occurred. In patients who experience incision infection, the repair surgery may be delayed by 3 or 6 months after the infection has resolved and the incision has healed. The mesh should be used with caution in the setting of emergency repair for incarcerated incisional hernia due to the higher risk of postoperative infection. Based on the location of the surgical mesh for strengthening the incisional hernia repair, the approach to incisional hernia repair may be divided as follows: (I) onlay/overlay method: a mesh is placed over the abdominal muscles; (II) sublay method: a mesh is placed under the abdominal muscles (or in the preperitoneal plane); and (III) intraperitoneal onlay mesh (IPOM)/underlay method: a mesh is placed next to the peritoneum. The underlay method is utilized in the laparoscopic incisional hernia repair, for which the antiadhesion mesh is required.

\section{Preoperative evaluation and preparation}

* The previous surgery history should be obtained, including the reasons for the previous surgery, surgical methods, postoperative diagnosis and incisional healing after surgery. In the case of recurrent incisional hernia, the details of the previous surgical repair should be obtained; 


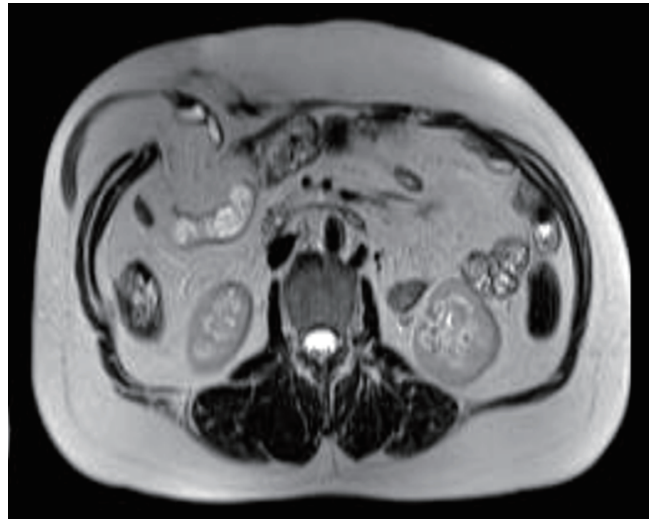

Figure 1 Magnetic resonance imaging (MRI) showing incisional hernia below the right costal margin.

* In patients with chronic cough and lung infection, secretion control and antibiotic treatment should be applied; the surgery may be scheduled beginning 1 week after the symptoms subside. All patients are advised to abstain from cigarettes for more than 2 weeks prior to surgery and to perform deep breathing training by thoracic extension exercises and diaphragmatic exercises;

* Obese patients should lose body weight before surgery and maintain a stable body weight after surgery;

* Abdominal computed tomography (CT) or magnetic resonance imaging (MRI) examinations are highly recommended. These examinations not only may be used to identify the location and size of the abdominal wall defect, the hernia contents and its relationship with its surrounding organs but also to calculate the volume ratio of the hernia sac volume to the peritoneal volume. To visualize the actual incisional hernia status, we recommend placing the patients in the lateral position and request that they hold their breath after a deep breath for the imaging examination. Additionally, comprehensive imaging examinations may help to assess tumor recurrence, metastasis or spread in the abdominal cavity in the tumor patient after surgery (Figure 1);

* Patients with moderate- to large-sized incisional hernia should wear a hernia support belt 2 weeks before surgery to adapt to daily activities. Patients with large incisional hernias should wear the hernia support belt 3 weeks before surgery and are advised to perform deep breathing training by thoracic extension exercises and diaphragmatic exercises to prevent postoperative dyspnea, pulmonary infection and abdominal compartment syndrome;
* A multidisciplinary treatment mode is recommended in patients with massive incisional hernias; the surgical plan should be determined by a multi-disciplinary team of specialists in plastic surgery, pulmonary medicine and intensive care to prevent abdominal compartment syndrome.

\section{Anesthesia}

General anesthesia with endotracheal intubation.

\section{Surgical preparation}

The skin of the abdomen and the umbilicus should be prepared according to laparoscopic surgery requirements. Bowel preparation is performed 1 day prior to surgery. Preoperative prophylactic antibiotics are administered, and a gastrointestinal decompression tube and a urethral catheter are placed 30 minutes before surgery.

\section{Surgical positioning}

Patients may be placed in the supine position. The operating table may require appropriate adjustment during surgery to facilitate exposure of the operative field.

\section{Surgeon location}

Monitors may be placed on the patient's affected side. The surgeon and the surgical assistant stand at the opposite side. An additional incision for the trocar may be made on the affected side if necessary. The surgeon may switch locations with the surgical assistant at any time as appropriate. The anesthetist stands at the patient's head. The instrument nurses stand on the same side as the surgeon.

\section{Trocar location}

Based on the location of the incisional hernia, the trocar location may be altered accordingly. Typically, trocars are placed along the bilateral anterior axillary lines or on the curved connecting line between the bilateral anterior superior iliac crests and the superior pubic symphysis (Figure 2). The first $12 \mathrm{~mm}$ trocar is used as a port for the laparoscope and mesh placement (an optical trocar may be superior in terms of safety). Under laparoscopic guidance, two $5-\mathrm{mm}$ trocars are placed. The locations of the three trocars are chosen according to the surgeon's preference. 
Page 4 of 8

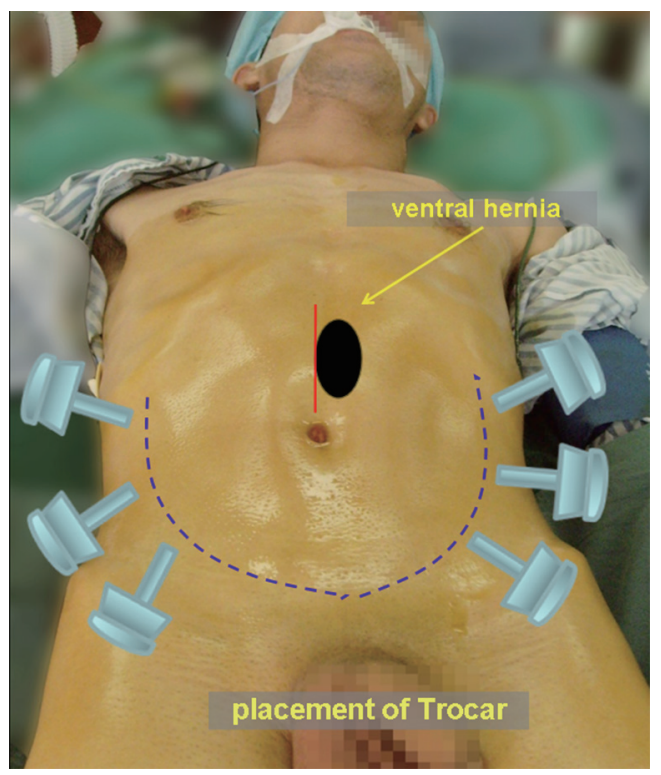

Figure 2 Placement of trocar.

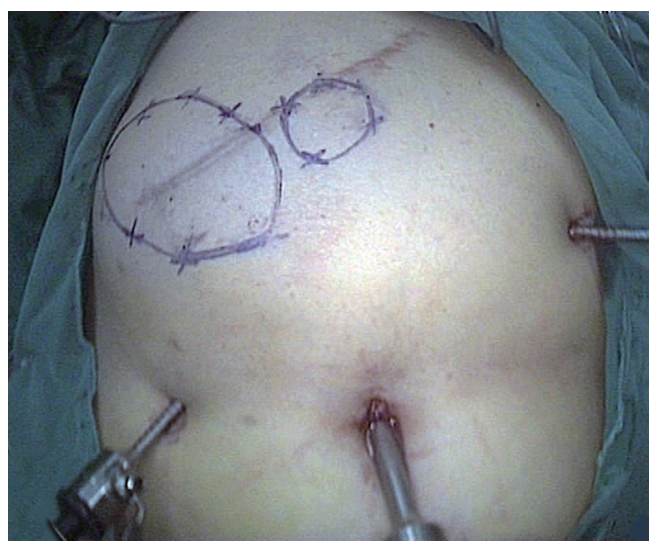

Figure 3 Trocar locations below the right costal margin for incisional hernia repair.

However, to avoid surgical instrument interference, the distance between the two $5-\mathrm{mm}$ trocars should be greater than $15 \mathrm{~cm}$.

\section{Surgical procedure}

* Preparation: the surgical area is routinely prepared and draped. A $1.5-\mathrm{mm}$ skin incision is made below the left costal margin (to reduce the risk of postoperative abdominal adhesions). A Veress needle is inserted into the abdominal cavity to create a pneumoperitoneum, and the pressure is maintained at $12-14 \mathrm{mmHg}$. Then,
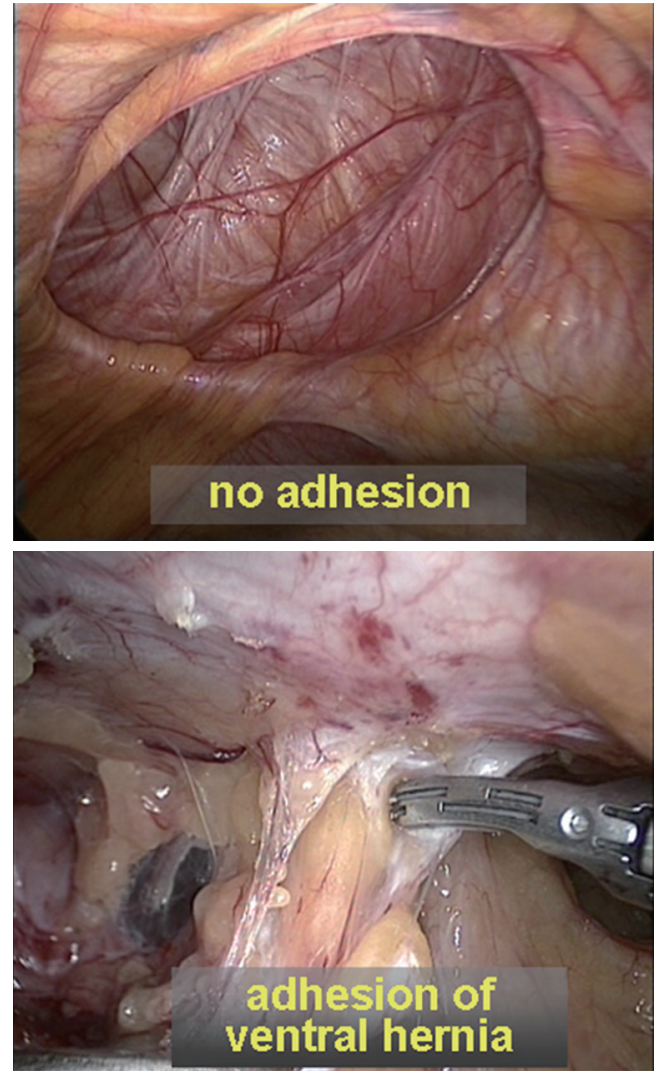

Figure 4 Views of the abdominal incision and hernia ring.

the first trocar is placed. The first trocar should be $5 \mathrm{~cm}$ or more from the original incision. The visceral sliding test with B-mode ultrasound or MRI examinations might be used to determine the location of milder abdominal adhesions for the placement of the first trocar. A $30^{\circ}$ laparoscope is inserted to explore the abdominal cavity and guide the placement of the other two 5-mm trocars (Figure 3);

* Separation of adhesions: the fascia or muscles surrounding the hernia ring (within 3-5 cm) are exposed. The operation is relatively easy in the few cases with a smooth hernia ring and absent adhesions (Figure 4). In contrast, in the presence of adhesions, scissors or an ultrasonic knife are required to separate them. The path of separation should be close to the abdominal wall. Sharp separation should be performed as much as possible: scissors separation (not using electricity) is appropriate for adhesions close to the bowel loops; coagulation may be performed only at the definite bleeding sites. When the separation of dense adhesions is highly difficult, the parietal peritoneum should be 


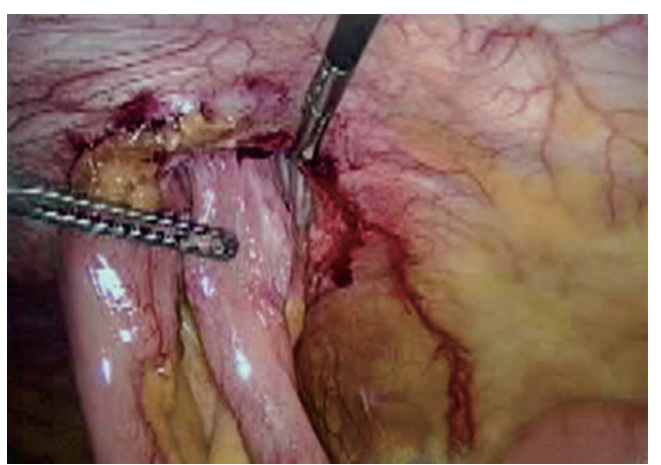

Figure 5 Dense adhesions between the bowel loops and the incisional hernia ring.

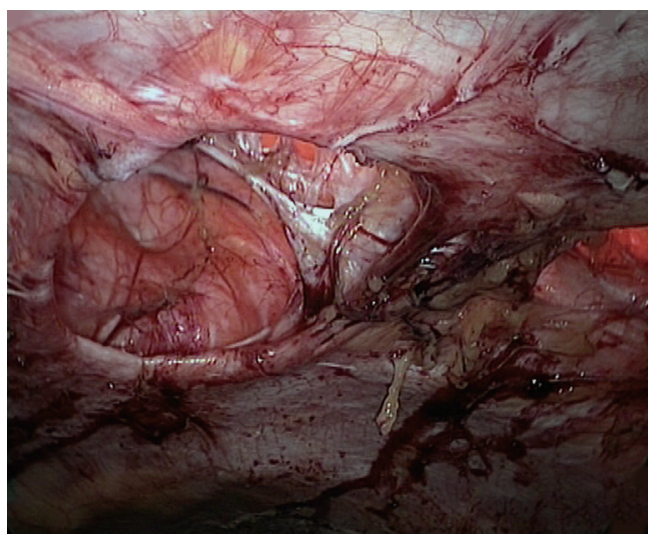

Figure 6 Incisional hernia defects below the right costal margin.

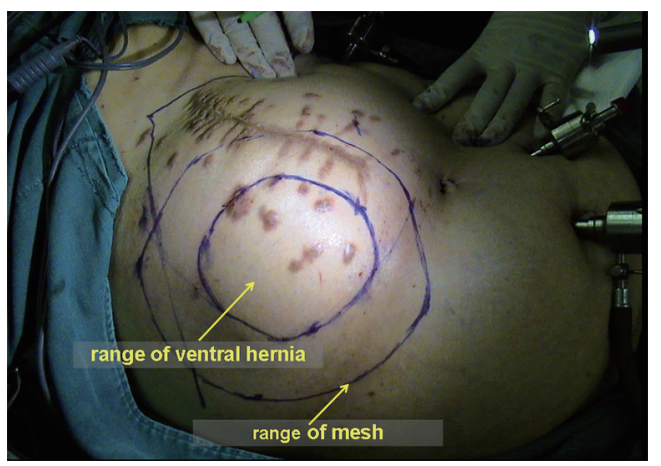

Figure 7 Marks for the abdominal wall defect and the mesh coverage.

incised and the partial abdominal wall with adhesions of the bowel loops should be separated en bloc to avoid damage to the bowel loops. The abdominal wall should be carefully examined after the adhesion separation to avoid overlooking occult abdominal wall defects. When the laparoscopic separation of adhesions around the hernia ring is difficult (Figure 5), particularly in the presence of dense adhesions between the contents (bowel loops) in the hernia sac and the ventral hernia coverings, pneumoperitoneum may be halted under laparoscopic light guidance, after which the hybrid techniques should be employed. Thereby, a spindle-shaped incision may be performed to remove the previous surgical wound scar and the hernia sac opened to remove the adhesive omentum. The proximal omentum and the bowel loops may be returned to the abdominal cavity. Complete separation between the hernia ring and the abdominal wall should be achieved as much as possible; after that, this incision may be closed, and the laparoscopic repair may continue;

* Defect and mesh size assessments: the extent of the abdominal wall defect and the mesh size has been determined, and the incised fascia has been closed. The adhered tissues $5 \mathrm{~cm}$ or more beyond the incisional hernia defect should be separated appropriately (Figure 6). A ureteral stent or sterile soft ruler is used to determine the defect size; the location of the hernia ring and the proposed mesh coverage are marked on the abdominal wall. After the pneumoperitoneum pressure is reduced by half, the location and the edge of the abdominal wall defect (including small occult abdominal wall defects) are marked by syringe needles inserted through the abdominal wall. The mesh size might be determined based on the defect edge plus a $4-\mathrm{cm}$ extension. The mesh coverage is also marked (Figure 7). EndoClose needles with non-absorbable suture are used to close the fascial defect by intermittent or continuous suturing through the full thickness of the abdominal wall via the small skin incision of the incisional hernia (Figure 8). If the spiral tacks with full-thickness suture of the abdominal wall are used to fixate the mesh, the suture point should be located at sites $1 \mathrm{~cm}$ beyond the mesh rim. These suture points should be evenly distributed along the mesh rim. The $6-8$ points should be marked on the abdominal skin, including the central mesh site;

* Preparation of the mesh: first, the surgical gloves must be changed. Then, the proper size of the anti-adhesion mesh is selected, and 6-8 sutures are set on the rough side (non-anti-adhesion side) along the rim of the mesh and at its central portion using 2-0 non-absorbable sutures. Equal-length double suture tails $15-20 \mathrm{~cm}$ long 


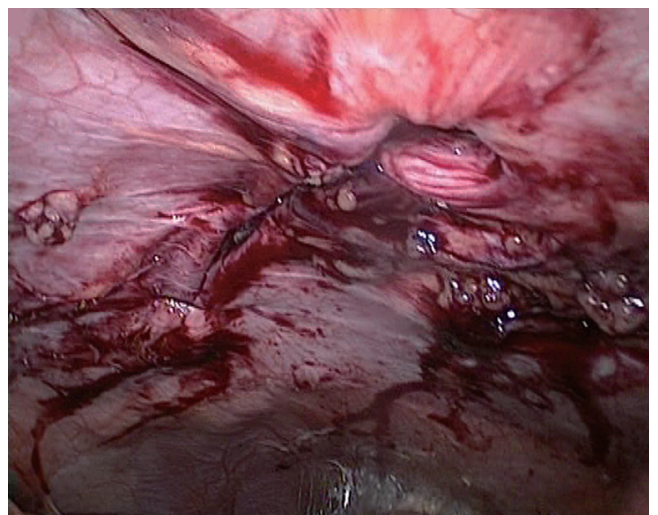

Figure 8 Interrupted sutures to close the incisional hernia fascial defect.

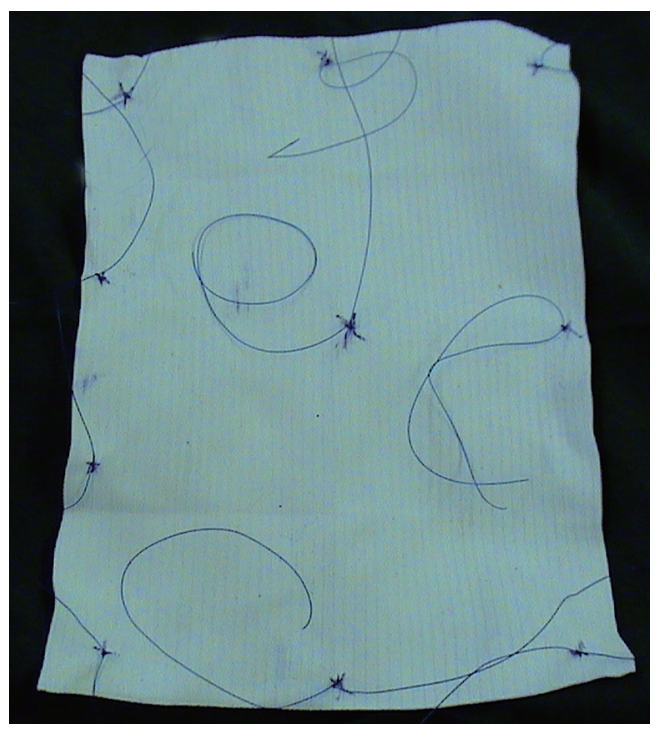

Figure 9 Preparation of the mesh.

should be left at each suture; then, the mesh is rolled tightly with the suture tails inside. To facilitate fast and accurate placement of the mesh, a suture may be preset in the center of the mesh and introduced out of the abdominal cavity via the center of the abdominal wall defect (Figure 9);

* Mesh placement and fixation: the rolled mesh is placed into the abdominal cavity through the $12-\mathrm{mm}$ trocar port and opened with the anti-adhesion side toward the abdominal cavity; the preset sutures are introduced out of the abdominal cavity using EndoClose needles or the puncture needles that pass through the full thickness of the abdominal wall via the predetermined sites. At each predetermined site, two needle punctures are made to ensure that the suture ends do not emerge from the same site. All the preset sutures are pulled appropriately to open the mesh and ensure that the mesh tightly covers the abdominal wall defect; the $5-\mathrm{mm}$ spiral tacks are used to fixate the mesh to the abdominal wall along the hernia ring and the mesh rim at intervals of $2-4 \mathrm{~cm}$. The pneumoperitoneum pressure is reduced by half; all sutures are tied (avoiding over-tying), and the knots are buried beneath the skin. International experts agree that to reduce the operative time and postoperative wound pain, the spiral tacks alone may be used for fixation of the mesh if the diameter of an incisional hernia defect is less than $5 \mathrm{~cm}$, whereas spiral tacks combined with full-thickness suture of the abdominal wall would be used for a hernia diameter defect greater than $5 \mathrm{~cm}$. For certain special incisional hernia locations, such as suprapubic and those below the costal margin, the mesh often cannot be directly fixated to the abdominal wall on one side of the defect. For suprapubic incision hernia repair, the lower rim of the mesh may be fixated to the pectineal ligaments; for the costal margin incisional hernia, the upper rim of the mesh may be inserted into the gap below the diaphragm and above the liver after incising the falciform ligament and then may be fixated by intermittent sutures through the full thickness of the abdominal wall at the lower edge of the costal margin;

* Drainage tube placement: an abdominal drainage tube is not routinely placed but is employed instead for postoperative observation in patients with extensive intraoperative separation or bowel loop repair. The drainage tube is not routinely placed between the mesh and the hernia sac. Its removal timing depends on the daily drainage volume and the properties of the drained fluid. The tube may be removed when the drainage volume is less than $5 \mathrm{~mL} /$ day;

* Conversion timing: the conversion to open surgery should be promptly determined in cases with extensive dense adhesions between the bowel loops in addition to intra-abdominal angular-shaped adhesions between the bowel loops and the abdominal wall or failure in the placement of the trocars and surgical instruments or when, to ensure the patient's safety, the laparoscopic separation of dense adhesions cannot be achieved;

* Postoperative care: the hernia support belt should be worn immediately after surgery for 3 months to ensure complete healing of the incision and the mesh. Early postoperative strenuous activities and heavy physical 
labor are prohibited.

\section{Management of complications}

* Postoperative abdominal distention and incomplete intestinal obstruction: abdominal distension with decreased bowel sounds are usually present 2 days after surgery and abdominal $\mathrm{X}$-ray examination often shows extensive intestinal dilations. The symptoms frequently subside spontaneously after 2 to 3 days. The prophylactic use of somatostatin for 2 to 3 days may inhibit intestinal fluid secretion and significantly reduce the incidence and degree of postoperative abdominal distention;

* Pain around the mesh area: notable pain is experienced when changing position or coughing. The degree of the pain is more severe in patients with full-thickness suturing of the abdominal wall combined with spiral tack fixation versus those with isolated spiral tack fixation. However, the pain is tolerable in most patients and does not require particular management except for analgesic use on postoperative days 1 to 2 . Occasionally, abdominal pain may last for 2 weeks and may be managed with oral anti-inflammatory agents;

* Seroma: a seroma located between the hernia sac and the mesh may be present 4 to 5 days after surgery. Seroma develops more often in patients with a large hernia sac with content adhesions or extensive intraoperative adhesion separation. Most seromas are absorbed spontaneously within 2 to 4 weeks after surgery; the fluid may be aspirated using strict aseptic techniques with ultrasound guidance if the seroma is large or persists for a long period. Repeated fluid aspiration and local compression with surgical dressings may resolve a seroma. The seroma occurrence rate may be reduced if the hernia volume is reduced by intermittent or continuous suturing during surgery or by making holes $(0.2 \mathrm{~cm})$ in the antiadhesion mesh with a sharp blade through which the fluid may leak into the abdominal cavity;

* Respiratory dysfunction: respiratory dysfunction mainly develops in patients with massive abdominal wall defects $(>12 \mathrm{~cm})$. Ventilator support may be required in severely affected patients. Sufficient abdominal cavity expansion before surgery (including wearing a hernia support belt and artificial pneumoperitoneum) may aid in preventing this complication;

* Bowl loop injury: intestinal perforation injury is common. Late-onset intestinal perforation may occur 2 or 3 days after surgery. The main causes include direct damage to the intestinal wall during the separation of adhesions or thermal injury from the instruments (delayed intestinal perforation). Therefore, the adhesion separation must be carefully performed to avoid damage to the intestinal wall. The identification of intraoperative injury to the seromuscular layer of the bowl loop requires immediate repair and drainage tube placement in the abdominal cavity. In cases of intraoperative injury through the fullthickness bowel loop wall, laparoscopic hernia repair should terminate after the bowel loop repair, and an abdominal drainage tube should be placed; laparoscopic hernia repair may be re-performed at 3 to 6 months after the injury. Some surgeons have reported performing same-stage repair of the injured intestinal wall and laparoscopic hernia repair if no severe contamination of the abdominal cavity caused by the intestinal content leakage had occurred. However, doctors and patients would both incur a significant risk of mesh infection. Should the bowel loop injury fail to be identified during surgery, early abdominal laparotomy, intestinal repair, colostomy (if necessary), peritoneal drainage and removal of the contaminated mesh should be performed once the signs of intestinal perforation are observed. Postponing the incisional hernia repair to the phase II repair will be necessary;

* Incisional hernia recurrence: because most patients with abdominal incisional hernias have undergone multiple surgeries, they tend to have varying degrees of local muscle and fascia atrophy. Small occult fascial defects often occur around the hernia ring. Therefore, choosing a mesh size that is too small for the defect due to an underestimated defect area is the major cause of incisional hernia recurrence. Combined mesh fixation using spiral tacks with full-thickness suture may avoid postoperative hernia recurrence caused by forcible tearing of the spiral tacks. The routine use of a hernia support belt for 2 to 3 months not only may reduce abdominal pain at the mesh-covered area but also may promote healing of the mesh and the abdominal wall. In the event of recurrent incisional hernia, re-repair is required;

* Infection: surgical gloves should be routinely changed before handling the mesh to prevent wound infection or repair area infection. The mesh should be directly placed into the abdominal cavity without contact with other surgical instruments and the skin in the surgical field through the trocar port to reduce the risk of mesh 
contamination. Once the mesh is infected, the infected mesh must be removed, which ultimately results in surgical failure of the hernia repair;

* Trocar site hernia: also known as trocar incisional hernia, trocar site hernia is a unique incisional hernia occurring after laparoscopic surgery that is more commonly observed with the use of a $12-\mathrm{mm}$ trocar incision. The muscular fascia at the larger trocar incision site should be closed carefully to prevent trocar site hernia. This closure should be completed before the patients recover from anesthesia to prevent the bowel loops or the omentum from entering the trocar site because of increased abdominal pressure;

- Massive ascites and cirrhosis: in patients with massive ascites and cirrhosis, the repair surgery should be planned very carefully. Refractory ascites not only may hinder healing of the surgical wound and the mesh integration with the abdominal wall tissue but also may increase the likelihood of infection, leading to failure of the hernia repair. Therefore, hernia repair may be considered in these patients when the ascites has been resolved using conservative treatments. Should surgery be indicated, an intra-abdominal drainage tube should be placed to reduce the amount of ascites.

Cite this article as: Yang XF, Liu JL. Laparoscopic repair of abdominal incisional hernia. Ann Transl Med 2016;4(18):342. doi: 10.21037/atm.2016.09.13

\section{Acknowledgements}

Funding: Shenzhen government funding for scientific and technical research and development (JCYJ20140414092023238).

\section{Footnote}

Conflicts of Interest: The authors have no conflicts of interest to declare.

Informed Consent: Written informed consent was obtained from the patient for publication of this manuscript and any accompanying images.

\section{References}

1. Vorst AL, Kaoutzanis C, Carbonell AM, et al. Evolution and advances in laparoscopic ventral and incisional hernia repair. World J Gastrointest Surg 2015;7:293-305.

2. Al Chalabi H, Larkin J, Mehigan B, et al. A systematic review of laparoscopic versus open abdominal incisional hernia repair, with meta-analysis of randomized controlled trials. Int J Surg 2015;20:65-74. 